\title{
Die Rebellion der Lebenswelt
}

\author{
Christo Stojanov
}

Die Transformationsforschung ist durch die Dominanz der in der Tradition von T. Parsons konzipierten Modernisierungstheorie kennzeichnet. Diese Dominanz läßt vor allem auf den Umstand zurückführen, daß nach dem Zusammenbruch des staatssozialistischen Systems die Modernisierungstheorie Parson'scher Prägung einen wesentlichen Vorsprung vor ihrem großen Konkurrenten - dem Marxismus-Leninismus - gewonnen hat. Auch ihr Doppelstatus - als wissenschaftliches und politisches Programm zugleich - hat einen Anteil an der Gewährleistung dieses Vorsprungs. Der Einfluß dieses Konzepts läßt sich nicht zuletzt auf die Tatsache zurückführen, daß es die gesellschaftliche Dynamik mit Hilfe von Kontrastkategorien darstellt: prämodern vs. modern, Plan- vs. Marktwirtschaft, Parteidiktatur vs. Demokratie usw. Aus einer solchen Perspektive erschien die Transformation als eine Substitution des 'Schwarzen' durch das 'Weiße'. Die dadurch erzielte Reduktion der Komplexität vermittelt einen Optimismus hinsichtlich der Überwindung des Status quo und wirkt zugleich als ein Mobilisierungsmedium.

Mit anderen Worten: Der Hauptinhalt der postsozialistischen Transformation wurde in der Implementierung, Anpassung und Neubildung von Institutionen gesellschaftlichen Lebens nach dem Muster der westlichen modernen Gesellschaften gesehen. Als vor-gestelltes Ziel galt die Übernahme des 'Modells' entwickelter westlicher Gesellschaften, das sich in der Systemauseinandersetzung als überlegen erwiesen hatte. Diese Vorstellung vom Prozeß eines radikalen Wandels in den ehemaligen sozialistischen Gesellschaften Ost- und Ostmitteleuropas stellt eine Folgerung der o.a. theoretischen Tradition dar und läßt sich als 'Modell-Transfer'-Konzept bezeichnen. Es spielt eine prägende Rolle sowohl für die Konstitution der Transformationsforschung als auch für die Reformpolitik.

Der normative Charakter der o.a. theoretischen Tradition erlaubte nach dem Zusammenbruch des institutionellen Gerüstes des Staatssozialismus, dessen Modernisierungsdefizite zu diagnostizieren. So ließ sich feststellen, was in diesen Gesellschaften fehlte (was als Voraussetzungen für die Etablierung von Marktwirtschaft und Demokratie angesehen wurde), d.h. was diese nicht waren, worüber diese nicht verfügten, wodurch diese sich von den westlichen Industriegesellschaften unterschieden, jedoch nicht deren tatsächliche 
Charakteristika und Gegebenheiten ${ }^{1}$. Mit anderen Worten: Eine solche Betrachtungsperspektive braucht keinen Bezug auf empirische Forschungsergebnisse - es genügt, lediglich die aus der analytischen Erfassung der 'Dritte-Welt-Gesellschaften' bekannte 'Meßlatte' anzulegen. Auf dieser Grundlage konnte die Modernisierungstheorie die Entwicklungsrichtung empfehlen, die den ex-sozialistischen Gesellschaften die Überwindung ihrer 'Defizite' gewährleisten würde. Die daraus resultierenden Konsequenzen für die Transformationsforschung lassen sich zusammen mit T. Hanf und R. Kreckel als 'Defizitforschung'2 oder mit K. Müller - wegen des nur negativen Bezugs auf die vorfindliche Gesellschaft (vgl. Müller, 1995: 48) - als 'negative Soziologie der Transformation' zusammenfassen.

Eine der wesentlichen Konsequenzen dieser Herangehensweise bezieht sich vor allem darauf, daß im Staatssozialismus entstandenes kulturelles und soziales 'Kapital' entwertet wurde. Die individuellen und kollektiven Identitäten und Biographien sollten demzufolge abgewertet bzw. durch westliche 'Importprodukte' ersetzt werden. Das ist eine der zentralen Feststellungen von Dr. Lungwitz, der ich ganz und gar zustimme. Der Zusammenbruch des Systems ist jedoch keinesfalls mit einem Zusammenbruch der Lebenswelt gleichzusetzen. Dieses Kapital war jedoch alles, was die postsozialistischen Menschen in das neue System mitbringen konnten. Wenn sie darauf verzichtet hätten, wäre ihnen automatisch eine untergeordnete Position zugewiesen worden. Es ist aus dieser Sicht nachvollziehbar, daß sie um den Wert ihrer Machtressourcen gekämpft haben sollten. Dadurch wurde von Anfang an dieses Konfliktpotential unterschätzt, gar als 'Sozialismus-Nostalgie' ideologisch abgewertet. Grundsätzlich können wir davon ausgehen, daß die Lebenswelt ein stark unterschätzter Bereich sowohl der Transformationsforschung als auch der Reformpolitik war. Im Mittelpunkt des Interesses stand die Entmachtung der alten Funktionsträger, d.h. die Zerstörung des alten Systems; der Aufbau eines neuen wurde mit dem Transfer institutioneller Strukturen gleichgesetzt. $\mathrm{Ob}$ diese Zerstörung politisch-ideologisch oder ökonomisch motiviert war, ist offen.

Der Glaube an die magische Wirksamkeit des Institutionen-Transfers nach westlichem Muster verwandelt sich in einen Glauben an die 'magische Kraft der

Höchst wahrscheinlich wäre die Diagnose ähnlich ausgefallen, wenn die westlichen Industriegesellschaften aus der Sicht des theoretisch-ideologischen Selbstverständnisses des Staatssozialismus hätten bewertet werden sollen.

2 „Die Transformationsforschung geriet vor allem bei westlichen KollegInnen zur Defizitforschung, indem die je aktuellen Abstände des untersuchten Bereiches zu den Zuständen der eigenen Referenzgesellschaft (bei uns natürlich vor allem in der Bundesrepublik) ermittelt und als weiterhin aufzuholender Modernisierungsrückstand ausgewiesen wurden.“ (Hanf/Kreckel: 40) 
Benennungen', wenn nicht die Funktionsweise, d.h. die individuellen und kollektiven Akteure mit ihren Machtressourcen, in ihren Wechselwirkungen auf den einzelnen Handlungsfeldern im Mittelpunkt des Interesses stehen. Mit anderen Worten: Die durch die Verteilung und Umverteilung der Machtressourcen entstehenden Einzelordnungen im Rahmen des neuen Systems stellen ein zentrales Feld der Transformationsforschung dar.

Auch bei weitem nicht alle Unternehmen und beruflichen Qualifikationen ließen sich als 'Schrott' kennzeichnen. Diese Tatsache wurde durch mehrere empirische Forschungsergebnisse bestätigt - sowohl durch jene, auf die Herr Lungwitz Bezug nimmt als auch durch unsere Studien zu den betrieblichen Transformationsmustern in Polen, Ungarn und Bulgarien ${ }^{3}$. Mit anderen Worten, dieses Modell-Transfer-Konzept, das der Transformationspolitik zugrunde lag, implizierte ein 'Überrollen bzw. Nichtig-Machen' der ganzen staatssozialistischen Geschichte, mit der nicht nur ein System, sondern individuelle Schicksale und Biographien verbunden waren. Deshalb ist auch verständlich, daß als Konsequenz dieser Herangehensweise im Verlauf der Transformation das Vertrauen zwischen Ost und West beeinträchtigt wurde. Hinzu kommt die Intervention vieler westlicher Experten, die dieses Verfahren durch lehrbuchmäßige, einfache Rezepte zu legitimieren versuchten. Dadurch gerieten in den Mittelpunkt der individuellen und kollektiven Aktivitäten die Strategien zur Rettung der bestehenden eigenen Position. Insbesondere durch die fragwürdigen Erfahrungen mit westlichen 'Reform-Rezepten' läßt sich seit etwa Mitte der 90er Jahre eine Ernüchterung im Hinblick auf ihre Wirksamkeit und eine zunehmende Pragmatisierung im Handeln des Managements in den mittel-/osteuropäischen Gesellschaften diagnostizieren.

In diesem Zusammenhang ist es angebracht, folgendes hervorzuheben: Der Begriff der postsozialistischen Transformation impliziert, daß dieser Prozeß einen Gegenstand hat. Es handelt sich nämlich um die Umgestaltung des 'Erbes' des Staatssozialismus. Dieses Erbe beinhaltet sowohl materielle als auch symbolische Ressourcen, vor allem die bestehende Kultur, deren ReKombination einen wesentlichen Teil des Transformationsprozesses ausmacht. Reformpraktiken sowohl auf gesamtgesellschaftlicher als auch auf betrieblicher Ebene lassen sich nach den Mustern des Umgangs mit diesem Erbe unterscheiden. Hierbei handelt es sich vor allem um den Bereich, der sich als symbolische Ressourcen ${ }^{4}$ in den einzelnen Unternehmen charakterisieren läßt. Die Muster des Umgangs mit dem staatssozialistischen 'Erbe' stellen ein wesentliches Differenzierungsmerkmal der einzelnen im Entstehen begriffenen betrieblichen Ordnungen dar. Unsere Forschungsergebnisse legen die Annahme

3 Vgl. u.a.: Dittrich/Fürstenberg/Schmidt, 1997

4 „Die symbolische Macht ist eine Macht, die in dem Maße existiert, wie es ihr gelingt, sich anerkennen zu lassen, sich Anerkennung zu verschaffen..." (Bourdieu, 1992: 82) 
nahe, insbesondere die Transformationsmuster auf der betrieblichen Ebene, die auf Kontinuität setzten und die unter staatssozialistischen Bedingungen akkumulierten (auch symbolischen) Ressourcen einsetzten, seien erfolgreicher im Hinblick auf die innerorganisatorische Integration und die wirtschaftlichen Ergebnisse als diejenigen, die einen Bruch mit staatssozialistischen Geschichte bevorzugen. Die Letzteren basieren in der Regel auf einer ideologisch geprägte Mythologisierung des Marktes und des neuen Systems, wodurch die jeweilige Ordnung im Unternehmen legitimiert wird. Daß diese Legitimationsinstanzen immer noch ziemlich fragil sind, bedarf keiner besonderen Argumentation. Die erfolgreichen Muster stellen eine wesentliche Dimension der Ernüchterung, Pragmatisierung des Handelns des Managements dar. Auch durch die unterschiedlichen Umgangsmuster mit symbolischen Ressourcen entsteht eine Vielfalt von neuen Ordnungen, die keinesfalls in das vorgegebene 'offizielle Kochbuch' der Transformation passen.

Abschließend möchte ich ausdrücklich der Analyse von Herrn Dr. Lungwitz beipflichten: In der Tat wurde die Eigenart und die Komplexität der postsozialistischen Transformation in den offiziellen Programmen wie jenem 'Back to Europe' unterschätzt ${ }^{5}$. Der Kapitalismus ist nirgendwo 'nach Design' (D. Stark) entstanden - das wird gewiß auch in Mittel- und Osteuropa nicht der Fall. Diese Unterschätzung bezieht sich vor allem auf die Rolle der lebensweltlichen Phänomene im Transformationsprozeß, auf die Vielfalt der Verknüpfungen zwischen der Lebenswelt und dem neuen System in allen Handlungsbereichen. M. Crozier stellte fest ${ }^{6}$, daß sich Managementtheorie und Praxis noch immer nicht von den technokratischen Vorstellungen der klassischen Organisationstheorie und -soziologie befreit haben; ihnen zufolge sollte der Einfluß der Lebenswelt mit Hilfe technischer Lösungen minimiert werden. Diese Diagnose ließe sich auf die postsozialistische Transformation übertragen. Einen erfolgversprechenden Weg zur Lösung des genannten Problems sieht Crozier in der Einbeziehung der sozialwissenschaftlichen Forschung in die Praxis. Insbesondere in Phasen eines beschleunigten Wandels sollen sich Organisationsstudien auf die grundsätzliche Logik der Management- und Reformpraktiken konzentrieren. Dies bedeutet erstens, die hinter den

5 Eines der wesentlichsten Defizite der meisten theoretischen Ansätze zur Untersuchung von Revolutionen - so S. Eisenstadt - ist das Fehlen einer „Unterscheidung zwischen den Bedingungen, die $\mathrm{zu}$ allgemeiner Unzufriedenheit, $\mathrm{zu}$ unterschiedlichen Formen gewaltsamen kollektiven Handelns, zum Sturz der Regierungen und/oder zur Revolution führen, und jenen, die die weitreichende soziale Veränderungen bewirken. Tatsächlich konzentriert sich der größte Teil der Revolutionsforschung auf den auffälligsten Aspekt dieses Phänomens - die politische Sphäre - und vernachlässigt die systematische Untersuchung des Zusammenhangs zwischen Transformation des politischen Bereichs und Transformation anderer Bereiche der Gesellschaftsordnung." (Eisenstadt, 1982: 24)

Vgl.: Crozier, 1992: 131 
normativen Orientierungen dieser Praktiken stehenden sozialwissenschaftlichen Annahmen und ihre Beziehungen zu Managementmodellen analytisch zu rekonstruieren und zweitens, sich mit der Frage auseinanderzusetzen, inwiefern sie der aktuellen Situation angemessen sind. Diese Problemdefinition, die auch ich teile, ist der Grund für die Schlußfolgerung, daß der soziologischen Forschung bei der Erprobung neuer Transformationslösungen in allen Handlungsbereichen und der Ausarbeitung neuer Prinzipien sowie Denkweisen eine zentrale Bedeutung zukommen könnte und auch müßte.

\section{Literaturhinweise}

Bourdieu, Pierre (1992) Die verborgenen Mechanismen der Macht. Hamburg

Crozier, Michel (1992) Entsteht eine neue Managementlogik?, - in: Journal für Sozialforschung, 32. Jg., H. 2, S.131-140

Dittrich, Eckhard/Fürstenberg, Friedrich/Schmidt, Gert (Hrsg.) (1997) Kontinuität im Wandel. Betriebe und Gesellschaften Zentraleuropas in der Transformation. München und Mering

Eisenstadt, Shmuel Noah (1982) Revolution und die Transformation von Gesellschaften. Eine vergleichende Untersuchung verschiedener Kulturen. Opladen.

Hanf, Thomas/Kreckel, Reinhard (1996) Identitätsvorstellungen in Transformationsprozessen, - in: Hodenius, Birgit/Schmidt, Gert (Hrsg.) Transformationsprozesse in MittelostEuropa (Sonderheft 4 der Soziologischen Review). München, S. 39-60

Müller, Klaus (1995) Vom Postkommunismus zur Postmodernität? Zur Erklärung sozialen Wandels in Osteuropa, - in: Kölner Zeitschrift für Soziologie und Sozialpsychologie, Jg. 47, H. 1, S. 37-64

Stark, David (1994): Nicht nach Design. Rekombiniertes Eigentum im osteuropäischen Kapitalismus, - in: PROKLA 94 (Politik in Deutschland), Jg. 24, S.127-142 